\title{
Sequential Circulating Tumor Cell Counts in Patients with Locally Advanced or Metastatic Hepatocellular Carcinoma: Monitoring the Treatment Response
}

\author{
Kun-Ming Rau ${ }^{1,2,+} \oplus$, Chien-Ting Liu ${ }^{3,4,+}$, Yu-Chiao Hsiao ${ }^{5}$, Kai-Yin Hsiao ${ }^{3}$, Tzu-Min Wang ${ }^{5}$, \\ Wei-Shan Hung ${ }^{5}$, Yu-Li Su ${ }^{3,4}{ }^{\oplus}$, Wei-Ching Liu ${ }^{3}$, Cheng-Hsu Wang ${ }^{4,6}$, Hsueh-Ling Hsu ${ }^{5}$, \\ Po-Heng Chuang $\left.{ }^{7} \mathbb{(}\right)$, Ju-Chien Cheng ${ }^{8}$ and Ching-Ping Tseng ${ }^{5,9,10,11, *}$ \\ 1 Department of Hematology-Oncology, E-Da Cancer Hospital, Kaohsiung 824, Taiwan; \\ liu07822@ms57.hinet.net \\ 2 School of Medicine, College of Medicine, I-Shou University, Kaohsiung 824, Taiwan \\ 3 Division of Hematology-Oncology, Department of Internal Medicine, Kaohsiung Chang Gung Memorial \\ Hospital, Kaohsiung 833, Taiwan; m7155@cgmh.org.tw (C.-T.L.); kilu9818@gmail.com (K.-Y.H.); \\ yolisu@mac.com (Y.-L.S.); a0920365248@gmail.com (W.-C.L.) \\ 4 College of Medicine, Chang Gung University, Taoyuan 333, Taiwan; chwang@adm.cgmh.org.tw \\ 5 Department of Medical Biotechnology and Laboratory Science, College of Medicine, Chang Gung University, \\ Taoyuan 333, Taiwan; lian31602@hotmail.com (Y.-C.H.); sugarcane025017@gmail.com (T.-M.W.); \\ srhhung@gmail.com (W.-S.H.); snow_0502@hotmail.com (H.-L.H.) \\ 6 Division of Hematology/Oncology, Department of Internal Medicine, Keelung Chang Gung Memorial \\ Hospital, Keelung 204, Taiwan \\ 7 Department of Internal Medicine, China Medical University Hospital, Taichung 404, Taiwan; \\ poheng2@yahoo.com.tw \\ 8 Department of Medical Laboratory Science and Biotechnology, China Medical University, Taichung 404, \\ Taiwan; jccheng@mail.cmu.edu.tw \\ 9 Graduate Institute of Biomedical Sciences, College of Medicine, Chang Gung University, \\ Taoyuan 333, Taiwan \\ 10 Molecular Medicine Research Center, Chang Gung University, Taoyuan 333, Taiwan \\ 11 Department of Laboratory Medicine, Linko Chang Gung Memorial Hospital, Taoyuan 333, Taiwan \\ * Correspondence: ctseng@mail.cgu.edu.tw; Tel.: +886-3211-8800 (ext. 5202) \\ + These authors contributed equally to this work.
}

Received: 15 December 2019; Accepted: 8 January 2020; Published: 10 January 2020

\begin{abstract}
Hepatocellular carcinoma (HCC) is among the most common causes of cancer death in men. Whether or not a longitudinal follow-up of circulating tumor cells (CTCs) before and at different time points during systemic/targeted therapy is useful for monitoring the treatment response of patients with locally advanced or metastatic HCC has been evaluated in this study. Blood samples $(n=104)$ were obtained from patients with locally advanced or metastatic HCC $(n=30)$ for the enrichment of CTCs by a negative selection method. Analysis of the blood samples from patients with defined disease status $(n=81)$ revealed that those with progressive disease (PD, $n=37)$ had significantly higher CTC counts compared to those with a partial response (PR) or stable disease (SD; $n=44$ for $\mathrm{PR}+\mathrm{SD}, p=0.0002)$. The median CTC count for patients with PD and for patients with PR and SD was 50 (interquartile range 21-139) and 15 (interquartile range 4-41) cells/mL of blood, respectively. A longitudinal analysis of patients $(n=17)$ after a series of blood collections demonstrated that a change in the CTC count correlated with the patient treatment response in most of the cases and was particularly useful for monitoring patients without elevated serum alpha-fetoprotein (AFP) levels. Sequential CTC enumeration during treatment can supplement standard medical tests and benefit the management of patients with locally advanced or metastatic HCC, in particular for the AFP-low cases.
\end{abstract}


Keywords: circulating tumor cells; hepatocellular carcinoma; longitudinal follow-up; alpha-fetoprotein

\section{Introduction}

The absolute incidence and mortality of hepatocellular carcinoma (HCC) worldwide reported in 2018 was 841,080 and 781,631, respectively [1]. It is the second most common cancer and the most common cause of cancer-related deaths in men in Taiwan. Hepatitis B virus (HBV)/hepatitis C virus $(\mathrm{HCV})$ infection and alcohol abuse are among the major etiologies for liver diseases and cancer. The underlying etiology of HCC due to HBV and HCV infections in Taiwan has been reduced because of universal HBV vaccination and the recent development of highly effective anti-HCV agents [2,3]. However, there is a parallel increase in HCC related to nonalcoholic fatty liver disease [4].

Once HCC is diagnosed, the Barcelona Clinic Liver Cancer algorithm, which includes in classifying HCC patients, clinical variables associated with tumor burden, the degree of liver dysfunction, and the patient's symptoms, is used for disease staging and treatment allocation [5,6]. A number of strategies have been employed for the treatment of patients with HCC. Transplantation remains the best option, but the supply of donor organs is limited. Alternative curative treatments including resection, radiofrequency ablation (RFA) [7], and, potentially, systemic and targeted therapy may delay recurrence $[8,9]$. When patients experience recurrence following the initial local therapies or transplantation, another local therapy and/or systemic treatments are required to obtain the best possible outcome. Abdominal ultrasound, radiological imaging, and serum alpha-fetoprotein (AFP) levels are the most common methods for surveillance of the treatment response. Imaging by computed tomography (CT) scans exposes patients to radiation, while serum AFP levels are in the normal range in $15-30 \%$ of patients with HCC [10]. For patients who are in clinical remission, all of the above methods cannot detect early recurrence.

During cancer progression, the number of circulating tumor cells (CTCs) increases and correlates with tumor mass [11]. Effective therapies that reduce tumor burden usually correlates with a decrease in the number of CTCs. Monitoring the CTC count is valuable in scrutinizing the treatment response of cancer patients and should provide for better patient care and management [12-14]. The presence of CTCs in patients with HCC has been reported in several studies [15-17]. CTC counts have been analyzed in most of these studies at a specific time point without longitudinal follow-up concerning the number or the change in CTC counts. Whether or not serial CTC counts during the course of treatment provides an advantage for monitoring the treatment response and disease status of patients with HCC remains to be explored.

In this study, patients with locally advanced or distant metastatic HCC were recruited for a series of CTC enumeration using a negative selection platform PowerMag which allows efficient depletion of $\mathrm{CD}_{4} 5^{+}$leukocytes and isolation of viable and label-free CTCs even only one CTC is present in $4 \mathrm{~mL}$ of peripheral blood [18]. The CD45 leukocytes are depleted for 10-20 folds more efficient by the PowerMag platform than by the EasySep method (StemCell Technologies, Vancouver, BC, Canada). The recovery rates of CTCs are $77-82 \%$ and $46-62 \%$ when cancer cells were enriched from leukocyte suspension and whole blood, respectively. The number of cancer cells recovered by PowerMag was linearly correlated with the number of cancer cells in the blood [18]. By the PowerMag platform, the number of CTCs and the change in the CTC counts were analyzed and correlated with the disease status and treatment response of the patients. Performing a series of CTC enumeration in parallel with the current standard medical methods provide benefits for the clinical care of patients with locally advanced or metastatic HCC, in particular for patients without elevated serum AFP levels. 


\section{Experimental Section}

\subsection{Patients}

This study was approved by the Institutional Review Board of Chang Gung Memorial Hospital (approval ID: 104-9667B) and E-Da Hospital (approval ID: EMRP66107). The selection criteria of patients were (1) presence of histologically/cytologically or clinically documented diagnosis of locally advanced or metastatic HCC with either serum AFPs $\geqq 400 \mathrm{ng} / \mathrm{mL}$, presence of cirrhosis and/or history of chronic HBV or HCV infections, or morphological evidence such as characteristic hypervascular liver tumors on contrast CT scans or magnetic resonance imaging (MRI); (2) age was $>20$ years old; (3) advanced HCC, when the patient was ineligible or when the patient did not consent to receiving local invasive treatment (resection, chemoembolization, or RFA); (4) the presence of at least one measurable disease which was defined as a lesion that can be measured as $10 \mathrm{~mm}$ in at least 1 dimension with a spiral CT scan or MRI; (5) patients willingness to either accept intra-arterial chemotherapy (IA-CT), intravenous chemotherapy (IV-CT), or targeted therapy such as sorafenib or other systemic therapy such as thalidomide, for HCC; (6) a life expectancy of at least 12 weeks; and (7) patients willing to provide written informed consent to participate in the study. A total of 30 patients with HCC were prospectively enrolled in the Division of Hematology and Oncology, Department of Internal Medicine, Kaohsiung Chang Gung Memorial Hospital. All patients gave written informed consent before enrollment in the study.

\subsection{Enrichment and Isolation of CTCS}

Blood samples were drawn from eligible patients following designed study protocols at day zero (baseline) and at subsequent periods of time after the start of treatment. A PowerMag negative selection system was used to enrich CTCs as described previously $[18,19]$. Briefly, fresh whole blood samples $(4 \mathrm{~mL})$ were processed by lysis of red blood cells (RBC) with an RBC lysis buffer $(0.15 \mathrm{M}$ $\mathrm{NH}_{4} \mathrm{Cl}$ and $\left.10 \mathrm{mM} \mathrm{NaHCO}_{3}\right)$ at room temperature. The mixture was then centrifuged $(400 \mathrm{~g})$ at $10{ }^{\circ} \mathrm{C}$ for $10 \mathrm{~min}$. The cell pellets were washed and subsequently resuspended in $2 \mathrm{~mL}$ of culture medium to obtain all nucleated cells. To deplete CD45 ${ }^{+}$-leukocytes, a CD45 depletion cocktail (StemCell Technologies, Vancouver, BC, Canada) was mixed with the collected nucleated cells and incubated at room temperature for $15 \mathrm{~min}$ to label $\mathrm{CD} 45^{+}$-leukocytes. The sample was then loaded onto a PowerMag column to separate $\mathrm{CD} 45^{+}$-leukocytes from the other nucleated cells. The cell filtrate was then centrifuged $(400 \times g)$ for $10 \mathrm{~min}$ and the procedure was repeated four times to remove most of the leukocytes. The pelleted cells were subject to further analysis.

\subsection{Immunofluorescence Staining and CTC Counting}

For immunofluorescence staining, $\mathrm{CD} 45^{+}$-depleted cell filtrates were incubated with an anti-EpCAM antibody (Abcam Inc., Cambridge, England) in the presence of the DNA staining dye Hoechst 33,342 at room temperature in the dark for $1 \mathrm{~h}$ as described previously [18,19]. After several washes and centrifugation, the supernatants were removed and the cell pellets were resuspended. The Alexa Fluor 488-conjugated donkey anti-mouse antibody (Invitrogen Inc., Carlsbad, CA, USA) was added to the cell suspension. After incubation in the dark for $30 \mathrm{~min}$, the unbound antibody was removed and the complete cell aliquot was placed on a slide. The full immunofluorescent images were captured by fluorescence microscopy using an automated slide scanning platform (Zeiss Axiovert 200M, Oberkochen, Germany) followed by image analysis using an IN Cell Analyzer 1000 Cellular Imaging and Analysis System (GE Healthcare Life Sciences, Pittsburgh, PA, USA). EpCAM ${ }^{+}-\mathrm{CTC}$ were defined as the cells with intact nucleus staining (Hoechst-positive) and positive for EpCAM. 


\subsection{Measurement of Serum AFP}

Serum AFP levels were measured at the same time as the CTC counts. An Abbott G4-5539/R05 Kit was used for measuring serum AFP levels. Analytical procedures were performed as described by the manufacturer.

\subsection{Statistical Analysis}

The CTC counts for patients with progressive disease (PD) and patients with partial response (PR) and stable disease (SD) were compared using the Mann-Whitney test. Statistical analysis was performed using Prism 5.0. $p<0.05$ was considered statistically significant.

\section{Results}

\subsection{Basic Characteristics of Enrolled Patients}

Thirty informative patients with advanced or metastatic HCC were enrolled in this study. The basic characteristics of these patients are shown (Table 1). The median age was 64 (interquartile range 56-69). Of the patients enrolled in this study, $67 \%$ and $33 \%$ were male and female, respectively. The initial Tumor, Node, Metastasis (TNM) classification of the tumors from 30 patients with HCC was based on the American Joint Committee on Cancer, seventh edition. Eight patients at diagnosis were at stage I, four at stage II, seventeen at stage III, and one at stage IV, respectively. All patients were diagnosed with locally advanced HCC and/or extrahepatic metastasis at the time of enrollment in this study. All clinicopathologic parameters of patients were classified according to the chart records.

Table 1. Basic characteristics of patients enrolled in this study.

\begin{tabular}{cc}
\hline Characteristics & No. of Patients \\
\hline Total enrollment & 30 \\
Stage at the time of diagnosis & \\
I & 8 \\
II & 4 \\
IV & 17 \\
Age at enrollment: median (interquartile range) & 1 \\
Sex (M/F) & $64(56-69)$ \\
Hepatitis history & $20 / 10$ \\
1 HBV ${ }^{+}$only & \\
HCV ${ }^{+}$only & 11 \\
HBV ${ }^{+}$HCV & 13 \\
Non-B, non-C & 2 \\
Tumor status at enrollment & 4 \\
Locally advanced & \\
Extrahepatic metastasis & 7 \\
Both & 9 \\
Treatment at enrollment & 14 \\
Sorafenib & \\
Ramucirumab & 21 \\
Nivolumab & 2 \\
Thalidomide & 1 \\
IA-CT & 2 \\
IV-CT & 3 \\
\hline
\end{tabular}

${ }^{1} \mathrm{HBV}$, hepatitis B virus; $\mathrm{HCV}$, hepatitis $\mathrm{C}$ virus; IA-CT, intra-arterial chemotherapy; IV-CT, intravenous chemotherapy.

Twenty-one patients received sorafenib at enrollment of this study. During the course of treatment, six patients had their treatment changed to thalidomide $(n=2)$, ramucirumab $(n=1)$, sorafenib $(n=1)$, IA-CT $(n=1)$, or IV-CT $(n=1)$. Nine patients who did not take sorafenib were treated with ramucirumab $(n=2)$, nivolumab $(n=1)$, thalidomide $(n=2)$, IA-CT $(n=3)$, or IV-CT $(n=1)$. 
Sorafenib was the standard treatment of patients with locally advanced or metastatic HCC in our institute and was prescribed from $400 \mathrm{mg}$ to $800 \mathrm{mg}$ daily based on the performance status and predicted tolerability of patients. The dosage was adjusted according to the side effects of sorafenib, with a maximum dose of $800 \mathrm{mg}$ daily. The regimen for IA-CT included doxorubicin and cisplatin [20]; the regimen for IV-CT was oxaliplatin and fluorouracil [21]. CTC counts every 2-3 months and the measurement of serum AFP levels was performed for monitoring the therapeutic response. Response Evaluation Criteria in Solid Tumors 1.1 (RECIST 1.1) was used for evaluating the therapeutic response according to the imaging study and the serum AFP level [22]. Disease status was defined as PD, SD, and PR, respectively.

\subsection{CTC Counts are Correlated with Disease Status of Patients with HCC}

A total of 104 blood samples were collected from patients during the course of chemo- or targeted-therapy (Table 2). The PowerMag negative selection system [18] was used to enrich CD45cells from peripheral blood by lysis of RBCs and depletion of CD45 $5^{+}$leukocytes. CTCs were defined as the EpCAM ${ }^{-}$positive cells in the $\mathrm{CD}_{4} 5^{+}$-depleted cell filtrates (Figure 1). The disease status for patients at each blood collection was determined and recorded based on an imaging study and the serum AFP level. Whether or not the disease status of patients correlated with the CTC count at that particular time point was analyzed. Thirty-seven occasions for all patients were defined as PD and 44 occasions were defined as PR $(n=18)$ or SD $(n=26)$. CTC counts for patients with PD were significantly higher than patients with PR or SD $(p=0.0002)$. The median CTC count for patients with PD and patients with PR + SD was 50 (interquartile range 21-139) and 15 (interquartile range 4-41) cells $/ \mathrm{mL}$ of blood, respectively (Figure 2). CTC counts facilitate the differentiation of patients with PD from patients with PR or SD.

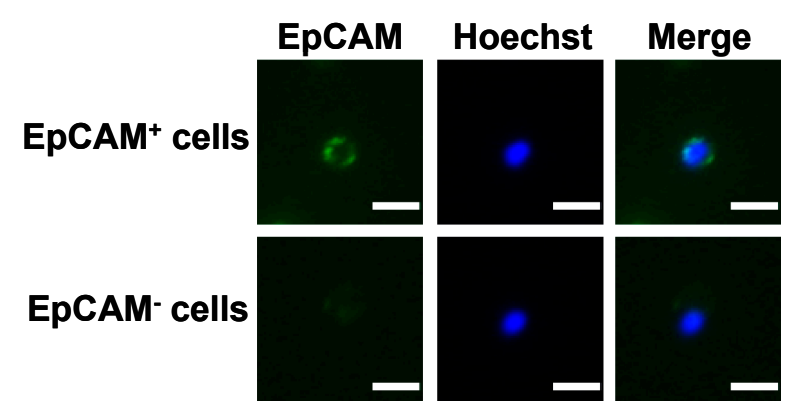

Figure 1. Schematic representation for the fluorescence images of $\mathrm{EpCAM}^{+}$and $\mathrm{EpCAM}^{-}$cells from individuals with advanced or metastatic HCC. The $\mathrm{CD} 45^{+}$-depleted cell populations that were positive and negative for EpCAM were defined, respectively. Positive staining of Hoechst 33,342 (blue) indicates the presence of intact nucleated cells. Bar $=10 \mu \mathrm{m}$. 
Table 2. The testing results for circulating tumor cells (CTCs) and alpha-fetoprotein (AFP) and the disease status of patients with hepatocellular carcinoma (HCC).

\begin{tabular}{|c|c|c|c|c|c|c|c|c|c|c|c|}
\hline \multirow{2}{*}{ Patient ID } & \multirow{2}{*}{ Clinical Parameters } & \multirow{2}{*}{ Treatment } & \multicolumn{9}{|c|}{ No. of Blood Collection } \\
\hline & & & 1 & 2 & 3 & 4 & 5 & 6 & 7 & 8 & 9 \\
\hline \multirow[t]{2}{*}{1} & CTCs (cells/mL) & & 4 & 2 & 2 & 10 & 2 & 32 & 12 & 1 & \\
\hline & $\operatorname{AFP}(\mathrm{ng} / \mathrm{mL})$ & Sorafenib; thalidomide after the 2 nd blood collection & 4737 & 47,360 & - & - & 11 & $<2$ & $<2$ & $<2$ & \\
\hline \multirow[t]{3}{*}{2} & CTCs (cells/mL) & & 45 & 2 & 3 & 26 & 26 & 43 & & & \\
\hline & $\mathrm{AFP}(\mathrm{ng} / \mathrm{mL})$ & Sorafenib & 323 & 675 & 3356 & 4822 & 4171 & - & & & \\
\hline & Disease status & & - & SD & $\mathrm{SD}$ & $\mathrm{PD}$ & $\mathrm{PD}$ & PD & & & \\
\hline 3 & Disease status & & PR & SD & SD & SD & SD & PD & PD & $\mathrm{PD}$ & $\mathrm{PD}$ \\
\hline \multirow[t]{3}{*}{4} & CTCs (cells/mL) & & 21 & 66 & 179 & 129 & 8 & & & & \\
\hline & $\operatorname{AFP}(\mathrm{ng} / \mathrm{mL})$ & Sorafenib & 5222 & 12,929 & 16,727 & - & - & & & & \\
\hline & Disease status & & - & SD & SD & PD & PD & & & & \\
\hline \multirow[t]{2}{*}{5} & CTCs (cells/mL) & & 20 & 211 & & & & & & & \\
\hline & $\operatorname{AFP}(\mathrm{ng} / \mathrm{mL})$ & Sorafenib & 59,233 & - & & & & & & & \\
\hline \multirow[t]{3}{*}{7} & CTCs (cells/mL) & & 64 & & & & & & & & \\
\hline & $\operatorname{AFP}(\mathrm{ng} / \mathrm{mL})$ & Sorafenib & 542 & & & & & & & & \\
\hline & Disease status & & PD & & & & & & & & \\
\hline \multirow[t]{3}{*}{8} & CTCs (cells/mL) & & 88 & & & & & & & & \\
\hline & $\operatorname{AFP}(\mathrm{ng} / \mathrm{mL})$ & Sorafenib & 8535 & & & & & & & & \\
\hline & Disease status & & $\mathrm{PD}$ & & & & & & & & \\
\hline \multirow[t]{3}{*}{9} & CTCs (cells/mL) & & 43 & & & & & & & & \\
\hline & $\operatorname{AFP}(\mathrm{ng} / \mathrm{mL})$ & Sorafenib & 656 & & & & & & & & \\
\hline & Disease status & & PD & & & & & & & & \\
\hline \multirow[t]{3}{*}{10} & CTCs (cells/mL) & & 74 & 94 & 1148 & 315 & & & & & \\
\hline & $\operatorname{AFP}(\mathrm{ng} / \mathrm{mL})$ & Sorafenib & 146 & - & 231 & 543 & & & & & \\
\hline & Disease status & & - & PD & PD & PD & & & & & \\
\hline
\end{tabular}


Table 2. Cont

\begin{tabular}{|c|c|c|c|c|c|c|c|c|c|c|c|}
\hline \multirow{2}{*}{ Patient ID } & \multirow{2}{*}{ Clinical Parameters } & \multirow{2}{*}{ Treatment } & \multicolumn{9}{|c|}{ No. of Blood Collection } \\
\hline & & & 1 & 2 & 3 & 4 & 5 & 6 & 7 & 8 & 9 \\
\hline \multirow[t]{2}{*}{12} & CTCs (cells/mL) & & 198 & 18 & & & & & & & \\
\hline & $\operatorname{AFP}(\mathrm{ng} / \mathrm{mL})$ & Sorafenib & 12,222 & 26,771 & & & & & & & \\
\hline \multirow[t]{3}{*}{13} & CTCs (cells/mL) & & 42 & 615 & 902 & & & & & & \\
\hline & AFP (ng/mL) & Sorafenib & 421 & 390 & 596 & & & & & & \\
\hline & Disease status & & $\mathrm{SD}$ & SD & $\mathrm{PD}$ & & & & & & \\
\hline 14 & Disease status & & $\mathrm{PD}$ & & & & & & & & \\
\hline \multirow[t]{3}{*}{15} & CTCs (cells/mL) & & 36 & 22 & 266 & & & & & & \\
\hline & $\operatorname{AFP}(\mathrm{ng} / \mathrm{mL})$ & Sorafenib & 12 & 30 & 57 & & & & & & \\
\hline & Disease status & & - & SD & PD & & & & & & \\
\hline \multirow[t]{2}{*}{16} & CTCs (cells/mL) & & 13 & 16 & 5 & & & & & & \\
\hline & $\operatorname{AFP}(\mathrm{ng} / \mathrm{mL})$ & IA-CT & 27,621 & - & - & & & & & & \\
\hline \multirow[t]{3}{*}{18} & CTCs (cells/mL) & & 2 & 12 & & & & & & & \\
\hline & $\operatorname{AFP}(\mathrm{ng} / \mathrm{mL})$ & IV-CT & 6633 & 11,677 & & & & & & & \\
\hline & Disease status & & - & PD & & & & & & & \\
\hline \multirow[t]{3}{*}{19} & CTCs (cells/mL) & & 6 & 5 & 4 & 21 & 3 & & & & \\
\hline & $\operatorname{AFP}(\mathrm{ng} / \mathrm{mL})$ & Ramucirumab & 840 & 11 & 11 & 3 & - & & & & \\
\hline & Disease status & & - & PR & PR & PR & $\mathrm{PR}$ & & & & \\
\hline \multirow[t]{3}{*}{20} & CTCs (cells/mL) & & 12 & 310 & 15 & & & & & & \\
\hline & $\mathrm{AFP}(\mathrm{ng} / \mathrm{mL})$ & Nivolumab; sorafenib after the 2 nd blood collection & 2491 & 3513 & 4508 & & & & & & \\
\hline & Disease status & & - & $\mathrm{PD}$ & PR & & & & & & \\
\hline \multirow[t]{3}{*}{21} & CTCs (cells/mL) & & 6 & 16 & & & & & & & \\
\hline & $\operatorname{AFP}(\mathrm{ng} / \mathrm{mL})$ & Ramucirumab & 14 & 14 & & & & & & & \\
\hline & Disease status & & - & PD & & & & & & & \\
\hline
\end{tabular}


Table 2. Cont

\begin{tabular}{|c|c|c|c|c|c|c|c|c|c|c|c|}
\hline \multirow{2}{*}{ Patient ID } & \multirow{2}{*}{ Clinical Parameters } & \multirow{2}{*}{ Treatment } & \multicolumn{9}{|c|}{ No. of Blood Collection } \\
\hline & & & 1 & 2 & 3 & 4 & 5 & 6 & 7 & 8 & 9 \\
\hline \multirow[t]{3}{*}{24} & CTCs (cells/mL) & & 7 & 64 & 4 & 9 & & & & & \\
\hline & AFP (ng/mL) & Sorafenib & 41 & 42 & - & 73 & & & & & \\
\hline & Disease status & & - & PR & SD & PD & & & & & \\
\hline \multirow[t]{3}{*}{25} & CTCs (cells/mL) & & 7 & 39 & 7 & 4 & 15 & 17 & & & \\
\hline & AFP (ng/mL) & Sorafenib & 14 & - & - & - & 13 & 37 & & & \\
\hline & Disease status & & - & SD & SD & $\mathrm{SD}$ & SD & $\mathrm{SD}$ & & & \\
\hline \multirow[t]{3}{*}{26} & CTCs (cells/mL) & & 615 & 272 & 117 & & & & & & \\
\hline & AFP (ng/mL) & Sorafenib & 4 & - & 4 & & & & & & \\
\hline & Disease status & & - & SD & PR & & & & & & \\
\hline \multirow[t]{3}{*}{27} & CTCs (cells/mL) & & 125 & 149 & 173 & 54 & 5 & & & & \\
\hline & AFP (ng/mL) & Sorafenib; IA-CT after the 2 nd blood collection & 5 & 4 & 3 & - & - & & & & \\
\hline & Disease status & & - & PD & PR & PR & PR & & & & \\
\hline \multirow[t]{3}{*}{28} & CTCs (cells/mL) & & 16 & 18 & 1955 & 165 & & & & & \\
\hline & AFP (ng/mL) & Sorafenib & 1254 & 1188 & 1859 & 689 & & & & & \\
\hline & Disease status & & - & PD & PD & PR & & & & & \\
\hline \multirow[t]{3}{*}{29} & CTCs (cells/mL) & & 134 & 24 & & & & & & & \\
\hline & AFP (ng/mL) & Thalidomide & 16 & 29 & & & & & & & \\
\hline & Disease status & & - & PD & & & & & & & \\
\hline \multirow[t]{3}{*}{30} & CTCs (cells/mL) & & 15 & & & & & & & & \\
\hline & $\operatorname{AFP}(n g / m L)$ & Thalidomide & 67,658 & & & & & & & & \\
\hline & Disease status & & SD & & & & & & & & \\
\hline
\end{tabular}

${ }^{1} \mathrm{SD}$, stable disease; $\mathrm{PR}$, partial response; $\mathrm{PD}$, progressive disease. 


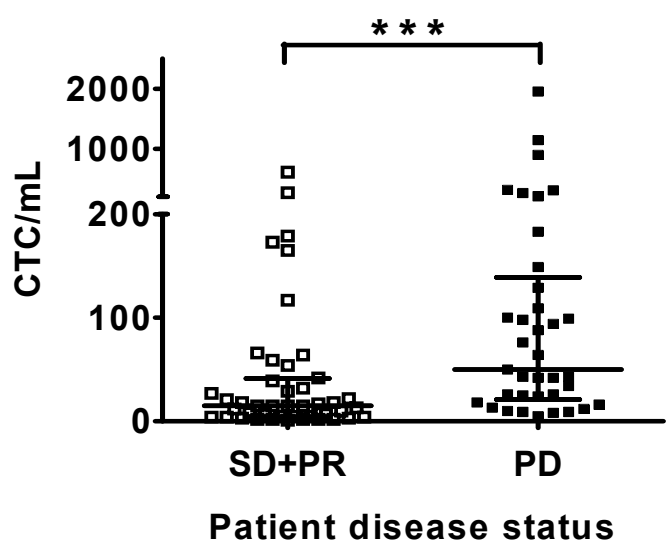

Figure 2. CTC counts for patients with progressive disease (PD), and patients with partial response (PR), or stable disease (SD). The disease status was determined at the time of blood collection for CTC counting. The CTC counts were plotted against the disease status of patients. A significant difference for the CTC counts was observed for the patients at the status of PR + SD and the patients at the status of PD. ${ }^{* * *}, p<0.0001$.

\subsection{Use of CTC Counts for Longitudinal Follow-Up and Disease Monitoring of Patients with HCC}

A total of 85 blood samples from 17 patients with at least three blood collections $(n=3-9)$ and two measurements of serum AFP levels $(n=2-8)$ were analyzed for investigating whether or not CTC counts are suitable for disease monitoring of patients with advanced or metastatic HCC. The data are presented in the following sections according to the serum AFP levels. The serum AFP of $100 \mathrm{ng} / \mathrm{mL}$ was used as a threshold to divide the patients according to a previous study by Hiraoka et al. who used AFP $>100 \mathrm{ng} / \mathrm{mL}$ to define positive cases for calculating the tumor marker score as a predictive prognosis value [23].

Patients \#1, \#17, and \#19 had an initial serum AFP $>100 \mathrm{ng} / \mathrm{mL}$ and a decreasing trend in the serum AFP levels during the course of the study. Among these three patients, patient \#1 was originally treated with sorafenib and was later changed to thalidomide after the second blood collection due to the side effects of sorafenib. Serum AFP was high at the first and second blood collection $(4737 \mathrm{ng} / \mathrm{mL}$ and $47,360 \mathrm{ng} / \mathrm{mL}$ ) when the disease status of the patient was considered as SD and it decreased to normal when the patient was at PR. CTC counts remained mostly low during the study period which was consistent with the clinical status of SD and PR (Figure 3A). Patient \#17 had a high serum AFP $(>500,000 \mathrm{ng} / \mathrm{mL}$ ) that decreased during follow-up. CTC counts remained high except for the second collection. The change of the CTC count was consistent with the disease status of the patient (Figure 3B). Patient \#19 received ramucirumab and underwent PR. Serum AFP (840 ng/mL) was decreased to normal after a series of treatments, while the CTC counts were low throughout the study (Figure 3C).

Patients \#2, \#4, \#10,\#13, \#20, \#22, and \#28 had an initial serum AFP $>100 \mathrm{ng} / \mathrm{mL}$ which increased during the course of study. Patient \#2 had an initial serum AFP of $323 \mathrm{ng} / \mathrm{mL}$ and was treated with sorafenib. The first two treatments appeared to be effective and the patient was at SD during the second and third blood collection. The serum AFP was increased and remained high $(675 \mathrm{ng} / \mathrm{mL}$ and $3356 \mathrm{ng} / \mathrm{mL}$ ) which predicted PD at the later time points. CTC counts decreased to below $10 \mathrm{cells} / \mathrm{mL}$ and increased as the disease progressed. The CTC count of this patient may be a reasonable marker of this patient's disease status (Figure 4A). Patient \#4 was treated with sorafenib and had a rise in the CTC counts and serum AFP levels from the first to the third blood collection. The patient was considered as having SD based on the imaging study. The patient's CTC count was decreased at the fifth blood collection and was not correlated with the disease status of PD. The patient died within a short period of time after the fifth blood collection. The decrease in the CTC count may reflect the intrinsic characteristics of this patient (Figure 4B). Patient \#10 received sorafenib but was unresponsive. The serum AFP levels continued to increase from the first to the fourth blood collection. The CTC 
count was decreased at the fourth blood collection but remained high throughout the period of study which was consistent with the disease status of PD (Figure 4C). Patient \#13 was at SD when enrolled in this study. Serum AFP levels remained high with a slight increase when the disease status changed from SD to PD during the second and third blood collections. The CTC count continued to rise, which was consistent with the change of disease status from SD to PD (Figure 4D). Patient \#20 was enrolled in a nivolumab trial. Serum AFP continued to increase. The patient's CTC count increased at the second blood collection. Treatment was changed to sorafenib and CTC count was decreased to 15 cells $/ \mathrm{mL}$ after the second blood collection. The patient's CTC count correlated with the change of disease status (Figure 4E). The initial elevation of the CTC count was likely a pseudo-progression, which is common at the beginning of immunotherapy [24]. Patient \#22 received IA-CT and reached SD after the initial treatment. The patient's serum AFP levels remained high during the same period of time and did not correlate with the patient's disease status, but the patient's CTC counts did (Figure 4F). Patient \#28 received sorafenib and had an initial serum AFP $>100 \mathrm{ng} / \mathrm{mL}$. The patient's CTC count correlated with the serum AFP level and the patient's disease status (Figure 4G).

A

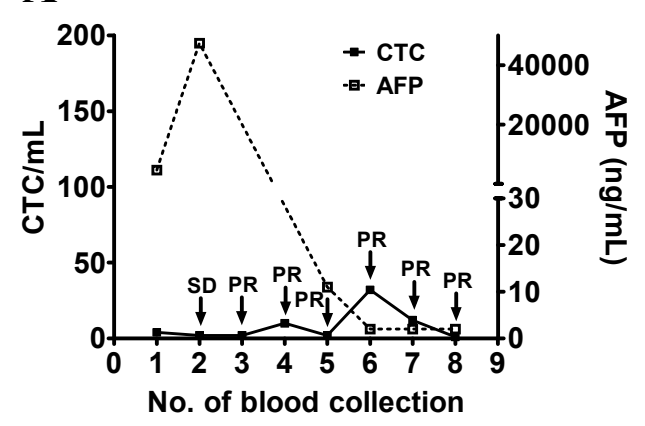

B

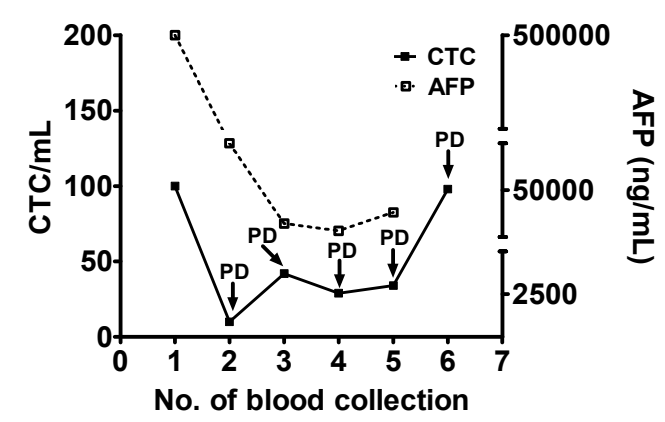

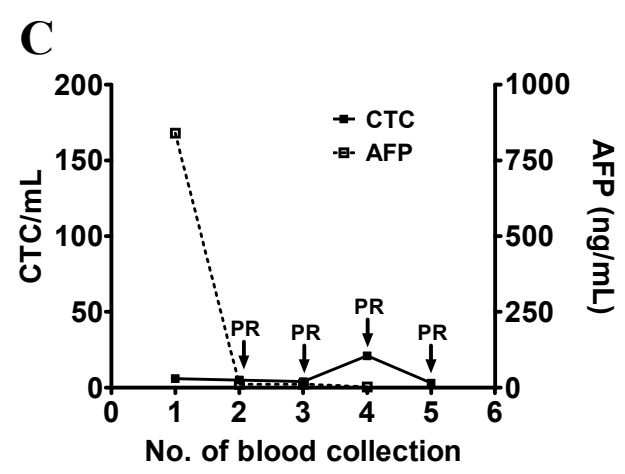

Figure 3. Longitudinal follow-up of the CTC counts for monitoring the disease status of patients with HCC (part I). (A-C) The blood samples were longitudinally collected from patients \#1 (panel A), \#17 (panel B), and \#19 (panel C), who had a high initial serum AFP level followed by a decrease. Series testing of the CTC count and the serum AFP level were performed and plotted accordingly. The disease status (SD, PR, and PD) was determined and was used as an indicator of the patient's clinical outcome.

Patients \#15, \#24, and \#25 had an initial serum AFP $<100 \mathrm{ng} / \mathrm{mL}$, which increased during the course of the study. Patient \#15 received sorafenib. The patient's serum AFP level increased slightly which predicted PD at the third blood collection. The change in the CTC count was consistent with the change of disease status from SD to PD (Figure 5A). Patient \#24 received sorafenib. The patient's serum AFP level remained high and the CTC counts correlated with the patient's disease status (Figure 5B). Patient \#25 received sorafenib. Both the serum AFP levels and the CTC counts remained steady throughout the study, which was consistent with the disease status of SD (Figure 5C). 
A

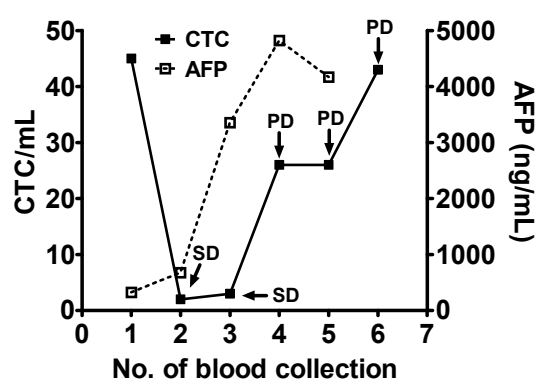

C

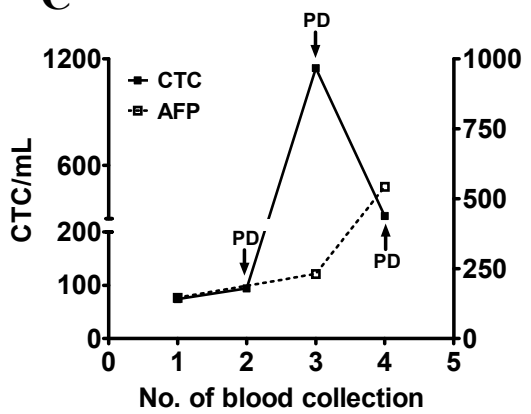

E

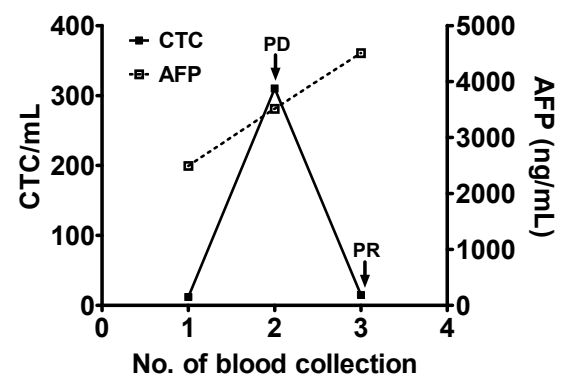

B

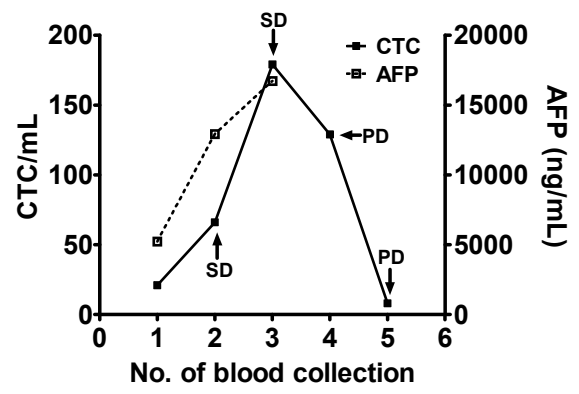

D

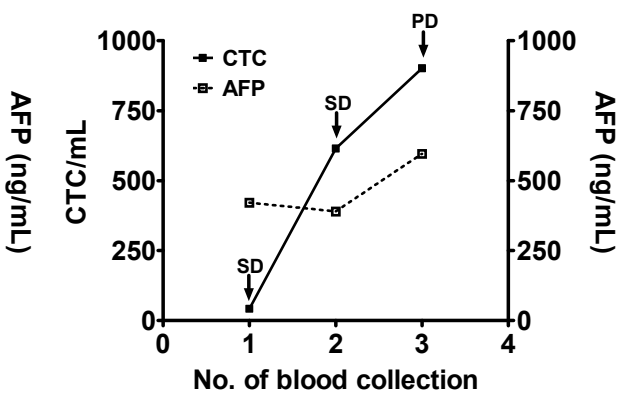

F

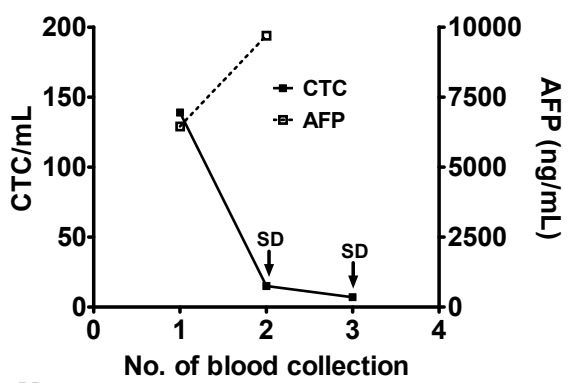

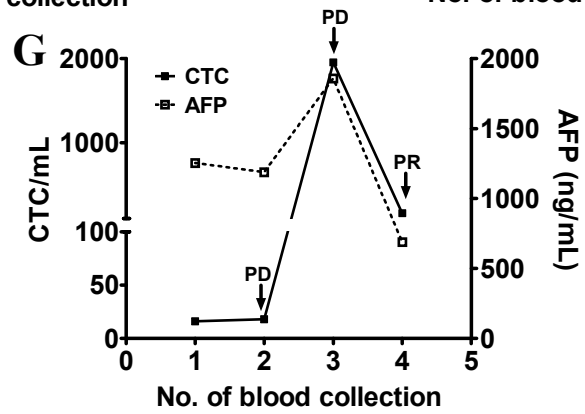

Figure 4. Longitudinal follow-up of the CTC counts for monitoring the disease status of patients with HCC (part II). (A-G) The blood samples were longitudinally collected from patients \#2 (panel A), \#4 (panel B), \#10 (panel C), \#13 (panel D), \#20 (panel E), \#22 (panel F), and \#28 (panel G), who all had a high initial serum AFP level followed by an increase. Series testing of the CTC count and serum AFP level were performed and plotted accordingly. The disease status (SD, PR, and PD) was determined and was used as an indicator of the patient's clinical outcome. 
A

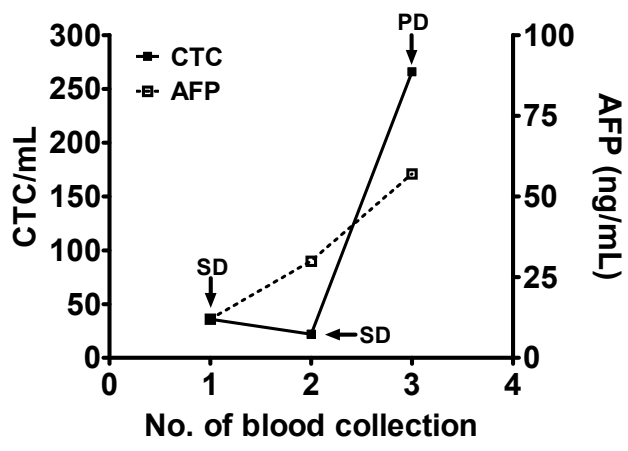

B

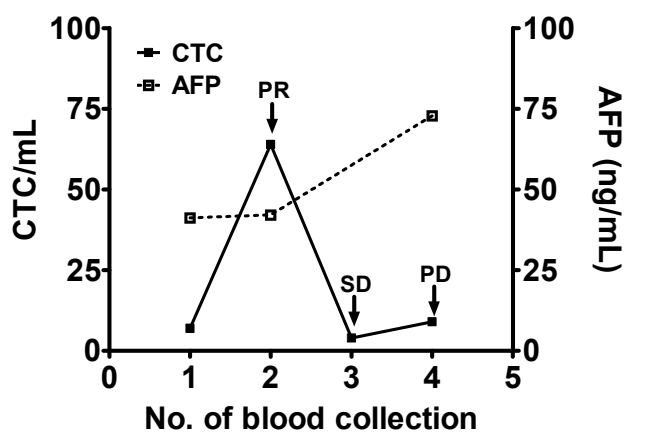

C

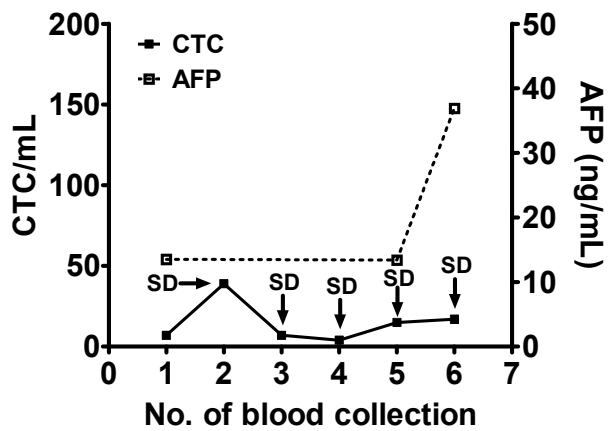

Figure 5. Longitudinal follow-up of CTC counts for monitoring the disease status of patients with HCC (part III). (A-C) The blood samples were longitudinally collected from patients \#15 (panel A), \#24 (panel B), and \#25 (panel C), who all had a moderate initial serum AFP level followed by an increase. Series testing of the CTC count and the serum AFP level were performed and plotted accordingly. The disease status (SD, PR, and PD) was determined and was used as an indicator of the patient's clinical outcome.

Patients \#3, \#23, \#26, and \#27 had an initial serum AFP $<100 \mathrm{ng} / \mathrm{mL}$ which remained steady during the course of study. Patient \#3 was originally treated with sorafenib and after the seventh blood collection was enrolled in a ramucirumab clinical trial. The serum AFP level was within normal range and did not correlate with the change of the patient's disease status. The CTC counts were mostly consistent with the patient's disease status (Figure 6A). Patient \#23 received sorafenib and was changed to IV-CT after the third blood collection. The serum AFP levels were steady throughout the study. The CTC counts were consistent with the change of the patient's disease status (Figure 6B). Patient \#26 received sorafenib treatment. The serum AFP levels remained low, which did not reflect the change of the patient's disease status. The patient's CTC counts decreased and was consistent with the disease status of SD and PR (Figure 6C). Patient \#27 received sorafenib during the period of the first and the second blood collection and was enrolled in the clinical trial receiving IA-CT thereafter. The serum AFP levels remained low and did not correlate with the patient's disease status. The CTC counts were high and were consistent with the disease status of PD at the second blood collection. The number of CTCs gradually decreased and was consistent with the change of disease status (Figure 6D). 
A

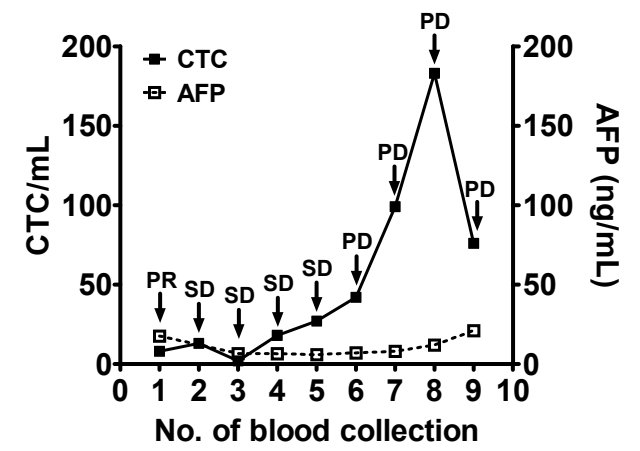

C

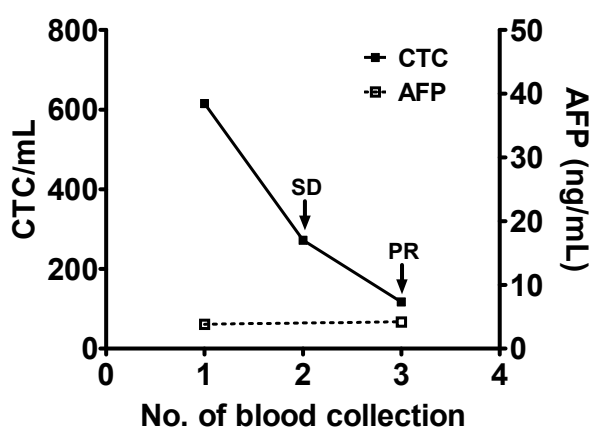

B

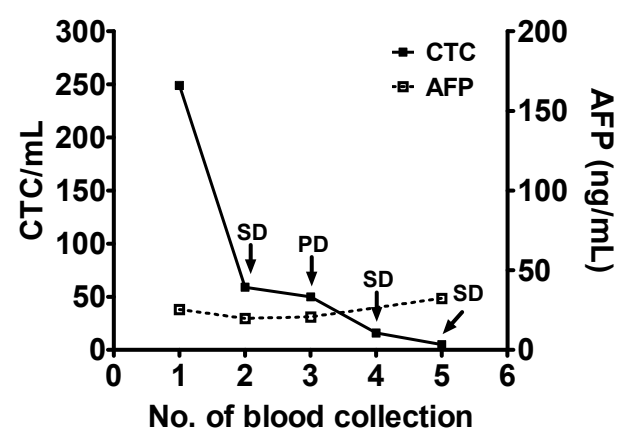

D

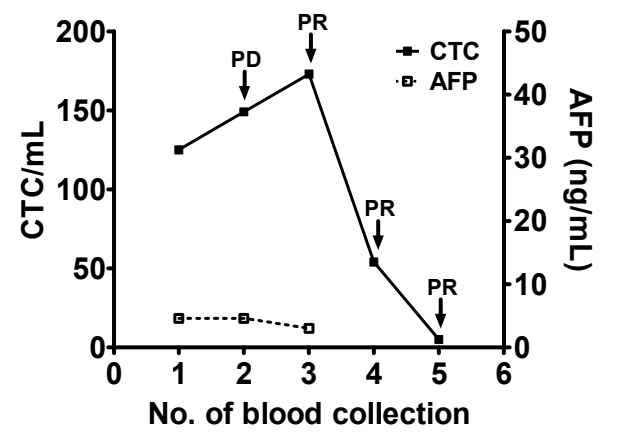

Figure 6. Longitudinal follow-up of the CTC counts for monitoring the disease status of patients with HCC (part IV). (A-D) The blood samples were longitudinally collected from patients \#3 (panel A), \#23 (panel B), \#26 (panel C), and \#27 (panel D), who all had a normal serum AFP level throughout the study. Series testing of the CTC count and the serum AFP level were performed and plotted accordingly. The disease status (SD, PR, and PD) was determined and was used as an indicator of the patient's clinical outcome.

\section{Discussion}

Monitoring disease status during treatment is crucial for the clinical management of patients with HCC. In the current study, the number of CTCs and the change in the CTC count are clinically significant and can supplement the use of serum AFP levels for evaluating the disease status of patients with HCC, in particular for the AFP-low cases.

Although serum AFP levels are routinely used in the clinical setting to monitor the disease status of patients with HCC, the serum AFP levels are found in the normal range for up to $42 \%$ of patients with HCC [25]. The use of serum AFP levels for HCC surveillance is not recommended by the American Association for the Study of Liver Diseases due to the lack of specificity [26]. Image examinations such as CT or MRI are still the standards for monitoring the treatment response. These procedures either need exposure to radiation or are expensive, and are not performed routinely. Novel HCC biomarkers such as des-gamma-carboxyprothrombin and lectin-bound AFP have been considered for overcoming the drawback of specifically using serum AFP levels for monitoring the treatment response of patients [27]. These markers exhibit poor sensitivity for HCC, particularly in the detection of lesions $<3 \mathrm{~cm}$ [28]. There is a need for additional markers for monitoring disease status and evaluating the treatment response in patients with HCC, in particular, those patients without elevated serum AFP levels.

With the heterogeneity and complexity of HCC tissue and cancer cells, real-time monitoring of cancer burden in response to therapy is important during the treatment course of patients with HCC. Although imaging study provides a direct assessment of cancer burden on the tissue, the intrinsic property of radiation makes it not suitable for routine longitudinal follow-up of patients. Serum 
biomarkers such as AFP offer an alternative avenue for real-time monitoring of cancer burden but with clear disadvantages in the clinical setting. Recent studies indicate that liquid biopsies are the suitable biological resources for longitudinal measurements of cancer burden and disease status. Liquid biopsies such as CTC and cell-free circulating tumor DNA offer the option of taking serial samples from a patient to detect changes during disease history and imposed by treatment [29]. Although serial monitoring of cell-free DNA was common, almost all reported CTC studies did only one to two measurements of CTC. For example, CTC counts have been used in previous studies for determining the diagnosis and assessing the prognosis of patients with HCC [15-17,30-32]. A correlation exists between the postoperative early recurrence of HCC and mesenchymal CTCs [32,33]. An elevated number of CTCs is associated with unfavorable clinicopathologic characteristics responsible for poor prognosis in patients with HCC [34]. An increase in the number of postoperative CTCs when compared to the number of preoperative CTCs is associated with lower survival and higher recurrence among patients with low AFP levels and cirrhosis [35]. Unlike prior studies, in which blood samples were collected at either a single or two time points prior to and after surgery or chemotherapy, blood samples in the current study were collected longitudinally before and during the course of systemic therapies. An evaluation of whether or not the number of CTCs or the change of CTC count related to the change of disease status in HCC patients was based on the CTC counts of each blood collection. With the continuous evolution of intrinsic cellular properties of HCC and the possible changes of therapeutic regimens during the treatment course, longitudinal follow-up of CTCs offers an option for real-time measurement of disease status for patients with HCC.

Several observations were made in the current study. First, CTCs were present in most of the blood samples collected from patients prior to systemic therapies. This is consistent with a number of HCC, papillary thyroid carcinoma, and head and neck cancer studies [19,36-38]. Second, patients at PD usually had higher CTC counts compared to patients at SD or PR. Although there is overlap for the two groups of patients, this may have minimal effect on the longitudinal measurements of CTC count and its correlation with patient disease status which is based on comparison to each patient's baseline CTC count. Further analysis of 85 blood samples from 17 patients with at least three sequential blood collections revealed that a change of the CTC count correlated with a change of disease status in most of the patients. The number of CTC or a change of the CTC count is not in accord with the clinical courses in several patients with high serum AFP, while in other cases AFP provides more useful clinical information and serves as a predictive biomarker even the CTC count reliably follows the clinical course of the patients. According to our analysis of patients who were AFP-low (Figure 6) but underwent disease progression, longitudinal measurements of CTC are informative and provide advantages for monitoring treatment response and disease status of patients without elevated serum AFP.

Simultaneous counting of CTCs and measurement of serum AFP levels are likely to provide more comprehensive information regarding the disease status and treatment response of patients with HCC. This is similar to our previous studies of papillary thyroid carcinoma [36,39]. The presence of CTC compromises serum thyroglobulin $(\mathrm{Tg})$ measurements when the anti-Tg antibody is present in patients with papillary thyroid carcinoma [39]. Combined analyses of CTC counts and serum Tg levels are superior to serum $\mathrm{Tg}$ levels alone in establishing the disease status of patients [36]. The combination of CTC counts with serum AFP levels and Tg should decrease false-negative rates and is recommended for disease monitoring of patients with the respective cancer types who are undergoing treatment. Because peripheral blood is easily accessible and provides an important biological resource for diagnosis and monitoring disease progression of cancer patients, combined analyses of CTCs and serum AFP levels with one blood draw can be performed more frequently than imaging and could pave the way for monitoring the disease status and treatment response of HCC patients.

The disease status of several patients was not correlated with the number of CTCs or the change of the CTC counts as discussed in the previous section. The reasons for these observations are not clear. The epithelial-mesenchymal transition (EMT) occurs during cancer progression. A loss or a decrease in EpCAM expression and an increase in mesenchymal markers such as $\mathrm{N}$-cadherin, vimentin, 
and podoplanin in cancer cells may accompany the EMT $[33,40,41]$. The number of EpCAM ${ }^{+}-\mathrm{CTC}$ is likely to decrease when the disease status of patients changes from SD to PD. This may result in a decrease in $\mathrm{EpCAM}^{+}-\mathrm{CTC}$ and an increase in $\mathrm{EpCAM}^{-}-\mathrm{CTC}$ counts when a patient's cancer is progressing. In the cell filtrate after depletion of $\mathrm{CD}_{4} 5^{+}$leukocytes, there are $\mathrm{EpCAM}^{-}$cells of which the number is variable in different blood samples from $10^{3}$ to $10^{4}$ cells. Usually, less than 5 cells in the cell filtrate were $\mathrm{CD}_{4} 5^{+}$[18]. Because $\mathrm{EpCAM}^{-}$cells are also present in the healthy control group, it is likely that most of the $\mathrm{EpCAM}^{-}$cells are $\mathrm{CD} 45^{-}$circulating endothelial cells or leukocytes such as apoptotic neutrophils [42,43], while some of the cells are EpCAM- ${ }^{-}$-CTCs. Future characterization of these $\mathrm{EpCAM}^{-}$cells should provide more insight into the clinical implication of monitoring this cell population. Consistent with this notion, different CTC subpopulations exist and elicit a distinct impact on the disease progression of patients. In this regard, the number of $\mathrm{CD} 133^{+} \mathrm{CD} 54^{+} \mathrm{CD} 44^{+}$circulating cancer stem cells is present in the blood and is a biomarker of treatment selection and liver metastasis in patients with CRC [44]. An RNA signature enables high specificity detection of CTCs in HCC [45]. Therefore, it is worthwhile to further characterize different CTC subsets and determine their respective impact on the disease status of HCC patients.

Sequential CTC counts during therapy can supplement serum AFP measurements and is able to provide timely information for monitoring treatment efficacy and clinical outcomes. The findings of the current study may have a significant impact on the clinical management of patients with locally advanced or metastatic HCC, in particular for patients without elevated serum AFP levels.

Author Contributions: Conceptualization, K.-M.R., C.-T.L., and C.-P.T.; data curation, Y.-C.H., C.-T.L., K.-Y.H., T.-M.W., H.-L.H., and P.-H.C.; data analysis, K.-M.R., Y.-C.H., W.-S.H., Y.-L.S., W.-C.L., J.-C.C., and C.-P.T.; investigation, K.-M.R., Y.-C.H., C.-T.L., W.-S.H., C.-H.W., P.-H.C., J.-C.C., and C.-P.T.; writing-original draft preparation, K.-M.R. and C.-P.T.; writing-review and editing, K.-M.R., C.-P.T., C.-T.L., and J.-C.C.; funding acquisition, K.-M.R., C.-P.T., C.-T.L., J.-C.C., and P.-H.C. All authors have read and agreed to the published version of the manuscript.

Funding: This work was funded by the Chang Gung Medical Foundation [grant numbers CMRPG8F1621-3, CMRPD1E0181-3, CMRPD1F0611-3, CMRPD1H0211-3, CMRPD1K0071, and BMRP466]; the Ministry of Science and Technology [grant number 105-2320-B-182-029-MY3, 106-2320-B-182-027-MY3, and 105-2314-B-182-042]; the Chang Gung Molecular Medicine Research Center [grant number EMRPD1G0081]; the China Medical University Hospital [grant number DMR-106-021 and CMU107-S-38], and the E-Da Cancer Hospital [grant number EDCHP108002] to K.-M.R., C.-T.L., C.-P.T., P.-H.C., and J.-C.C.

Acknowledgments: We appreciate Arnold Stern (New York University School of Medicine) for the editorial help in the preparation of this manuscript.

Conflicts of Interest: The authors declare no conflict of interest. The funders had no role in the design of the study; in the collection, analyses, or interpretation of data; in the writing of the manuscript, or in the decision to publish the results.

\section{References}

1. Bray, F.; Ferlay, J.; Soerjomataram, I.; Rebecca, L.; Siegel, R.L.; Lindsey, A.; Torre, L.A.; Jemal, A. Global cancer statistics 2018: GLOBOCAN estimates of incidence and mortality worldwide for 36 cancers in 185 countries. CA A Cancer J. Clin. 2018, 68, 394-424. [CrossRef] [PubMed]

2. Chang, M.H. Hepatitis B vaccination: Disease and cancer prevention-a Taiwanese experience. Clin. Liver Dis. 2010, 14, 521-530. [CrossRef] [PubMed]

3. Sulkowski, M.S.; Gardiner, D.F.; Rodriguez-Torres, M.; Reddy, K.R.; Hassanein, T.; Jacobson, I.; Lawitz, E.; Lok, A.S.; Hinestrosa, F.; Thuluvath, P.J.; et al. Daclatasvir plus sofosbuvir for previously treated or untreated chronic HCV infection. N. Engl. J. Med. 2014, 370, 211-221. [CrossRef] [PubMed]

4. White, D.L.; Kanwal, F.; El-Serag, H.B. Association between nonalcoholic fatty liver disease and risk for hepatocellular cancer, based on systematic review. Clin. Gastroenterol. Hepatol. 2012, 10, 1342-1359. [CrossRef]

5. Marrero, J.A.; Kulik, L.M.; Sirlin, C.B.; Zhu, A.X.; Finn, R.S.; Abecassis, M.M.; Roberts, L.R.; Heimbach, J.K. Diagnosis, staging, and management of hepatocellular carcinoma: 2018 practice guidance by the American Association for the Study of Liver Diseases. Hepatology 2018, 68, 723-750. [CrossRef] 
6. European Association for the Study of the Liver. EASL clinical practice guidelines: Management of hepatocellular carcinoma. J. Hepatol. 2018, 69, 182-236. [CrossRef]

7. Daher, S.; Massarwa, M.; Benson, A.A.; Khoury, T. Current and future treatment of hepatocellular carcinoma: An updated comprehensive review. J. Clin. Transl. Hepatol. 2018, 6, 69-78. [CrossRef]

8. Llovet, J.M.; Ricci, S.; Mazzaferro, V.; Hilgard, P.; Gane, E.; Blanc, J.F.; de Oliveira, A.C.; Santoro, A.; Raoul, J.L.; Forner, A.; et al. Sorafenib in advanced hepatocellular carcinoma. N. Engl. J. Med. 2008, 359, 378-390. [CrossRef]

9. Cheng, A.L.; Kang, Y.K.; Chen, Z.; Tsao, C.J.; Qin, S.; Kim, J.S.; Luo, R.; Feng, J.; Ye, S.; Yang, T.S.; et al. Efficacy and safety of sorafenib in patients in the Asia-Pacific region with advanced hepatocellular carcinoma: A phase III randomised, double-blind, placebo-controlled trial. Lancet Oncol. 2009, 10, 25-34. [CrossRef]

10. Hu, J.; Wang, N.; Yang, Y.; Ma, L.; Han, R.; Zhang, W.; Yan, C.; Zheng, Y.; Wang, X. Diagnostic value of alpha-fetoprotein combined with neutrophil-to-lymphocyte ratio for hepatocellular carcinoma. BMC Gastroenterol. 2018, 18, 186. [CrossRef]

11. Cabel, L.; Proudhon, C.; Gortais, H.; Loirat, D.; Coussy, F.; Pierga, J.Y.; Bidard, F.C. Circulating tumor cells: Clinical validity and utility. Int. J. Clin. Oncol. 2017, 22, 421-430. [CrossRef] [PubMed]

12. Olmos, D.; Baird, R.D.; Yap, T.A.; Massard, C.; Pope, L.; Sandhu, S.K.; Attard, G.; Dukes, J.; Papadatos-Pastos, D.; Grainger, P.; et al. Baseline circulating tumor cell counts significantly enhance a prognostic score for patients participating in phase I oncology trials. Clin. Cancer Res. 2011, 17, 5188-5196. [CrossRef] [PubMed]

13. Cristofanilli, M.; Budd, G.T.; Ellis, M.J.; Stopeck, A.; Matera, J.; Miller, M.C.; Reuben, J.M.; Doyle, G.V.; Allard, W.J.; Terstappen, L.W.; et al. Circulating tumor cells, disease progression, and survival in metastatic breast cancer. N. Engl. J. Med. 2004, 351, 781-791. [CrossRef] [PubMed]

14. Yap, K.; Cohen, E.N.; Reuben, J.M.; Khoury, J.D. Circulating tumor cells: State-of-the-art update on technologies and clinical applications. Curr. Hematol. Malig. Rep. 2019, 14, 353-357. [CrossRef] [PubMed]

15. von Felden, J.; Schulze, K.; Krech, T.; Ewald, F.; Nashan, B.; Pantel, K.; Lohse, A.W.; Riethdorf, S.; Wege, H. Circulating tumor cells as liquid biomarker for high HCC recurrence risk after curative liver resection. Oncotarget 2017, 8, 89978-89987. [CrossRef]

16. Zhou, J.; Huang, A.; Yang, X.R. Liquid biopsy and its potential for management of hepatocellular carcinoma. J. Gastrointest. Cancer 2016, 47, 157-167. [CrossRef]

17. Ogle, L.F.; Orr, J.G.; Willoughby, C.E.; Hutton, C.; McPherson, S.; Plummer, R.; Boddy, A.V.; Curtin, N.J.; Jamieson, D.; Reeves, H.L. Imagestream detection and characterisation of circulating tumour cells-a liquid biopsy for hepatocellular carcinoma? J. Hepatol. 2016, 65, 305-313. [CrossRef]

18. Lin, H.C.; Hsu, H.C.; Hsieh, C.H.; Wang, H.M.; Huang, C.Y.; Wu, M.H.; Tseng, C.P. A negative selection system PowerMag for effective leukocyte depletion and enhanced detection of EpCAM positive and negative circulating tumor cells. Clin. Chim. Acta 2013, 419, 77-84. [CrossRef]

19. Lin, J.D.; Liou, M.J.; Hsu, H.L.; Leong, K.K.; Chen, Y.T.; Wang, Y.R.; Hung, W.S.; Lee, H.Y.; Tsai, H.J.; Tseng, C.P. Circulating epithelial cell characterization and correlation with remission and survival in patients with thyroid cancer. Thyroid 2018, 28, 1479-1489. [CrossRef]

20. Ma, M.C.; Chen, Y.Y.; Li, S.H.; Cheng, Y.F.; Wang, C.C.; Chiu, T.J.; Pei, S.N.; Liu, C.T.; Huang, T.L.; Huang, C.H.; et al. Intra-arterial chemotherapy with doxorubicin and cisplatin is effective for advanced hepatocellular cell carcinoma. Sci. World J. 2014, 2014, 160138. [CrossRef]

21. Qin, S.; Cheng, Y.; Liang, J.; Shen, L.; Bai, Y.; Li, J.; Fan, J.; Liang, L.; Zhang, Y.; Wu, G.; et al. Efficacy and safety of the FOLFOX4 regimen versus doxorubicin in Chinese patients with advanced hepatocellular carcinoma: A subgroup analysis of the EACH study. Oncologist 2014, 19, 1169-1178. [CrossRef]

22. Schwartz, L.H.; Seymour, L.; Litière, S.; Ford, R.; Gwyther, S.; Mandrekar, S.; Shankar, L.; Bogaerts, J.; Chen, A.; Dancey, J.; et al. RECIST 1.1-standardisation and disease-specific adaptations: Perspectives from the RECIST Working Group. Eur. J. Cancer 2016, 62, 138-145. [CrossRef]

23. Hiraoka, A.; Michitaka, K.; Kumada, T.; Izumi, N.; Kadoya, M.; Kokudo, N.; Kubo, S.; Matsuyama, Y.; Nakashima, O.; Sakamoto, M.; et al. Prediction of prognosis of intermediate-stage HCC patients: Validation of the tumor marker score in a nationwide database in Japan. Liver Cancer 2019, 8, 403-411. [CrossRef]

24. Ma, Y.; Wang, Q.; Dong, Q.; Zhan, L.; Zhang, J. How to differentiate pseudoprogression from true progression in cancer patients treated with immunotherapy. Am. J. Cancer Res. 2019, 9, 1546-1553. 
25. Gomaa, A.I.; Khan, S.A.; Leen, E.L.S.; Waked, I.; Taylor-Robinson, S.D. Diagnosis of hepatocellular carcinoma. World J. Gastroenterol. 2009, 15, 1301-1314. [CrossRef]

26. Ahmed Mohammed, H.F.; Roberts, L.R. Should AFP (or any biomarkers) be used for HCC surveillance? Curr. Hepatol. Rep. 2017, 16, 137-145. [CrossRef]

27. Marrero, J.A.; Feng, Z.; Wang, Y.; Nguyen, M.H.; Befeler, A.S.; Roberts, L.R.; Reddy, K.R.; Harnois, D.; Llovet, J.M.; Normolle, D.; et al. Alpha-fetoprotein, des-gamma carboxyprothrombin, and lectin-bound alpha-fetoprotein in early hepatocellular carcinoma. Gastroenterology 2009, 137, 110-118. [CrossRef]

28. Lou, J.; Zhang, L.; Lv, S.; Zhang, C.; Jiang, S. Biomarkers for hepatocellular carcinoma. Biomark. Cancer 2017, 9, 1-9. [CrossRef]

29. Ye, Q.; Ling, S.; Zheng, S.; Xu, X. Liquid biopsy in hepatocellular carcinoma: Circulating tumor cells and circulating tumor DNA. Mol. Cancer 2019, 18, 114. [CrossRef]

30. Zhou, Y.; Wang, B.; Wu, J.; Zhang, C.; Zhou, Y.; Yang, X.; Zhou, J.; Guo, W.; Fan, J. Association of preoperative EpCAM circulating tumor cells and peripheral Treg cell levels with early recurrence of hepatocellular carcinoma following radical hepatic resection. BMC Cancer 2016, 16, 506. [CrossRef]

31. Kelley, R.K.; Magbanua, M.J.; Butler, T.M.; Collisson, E.A.; Hwang, J.; Sidiropoulos, N.; Evason, K.; McWhirter, R.M.; Hameed, B.; Wayne, E.M.; et al. Circulating tumor cells in hepatocellular carcinoma: A pilot study of detection, enumeration, and next-generation sequencing in cases and controls. BMC Cancer 2015, 15, 206. [CrossRef]

32. Chen, Y.; Li, S.; Li, W.; Yang, R.; Zhang, X.; Ye, Y.; Yu, J.; Ye, L.; Tang, W. Circulating tumor cells undergoing EMT are poorly correlated with clinical stages or predictive of recurrence in hepatocellular carcinoma. Sci. Rep. 2019, 9, 7084. [CrossRef]

33. Wang, Z.; Luo, L.; Cheng, Y.; He, G.; Peng, B.; Gao, Y.; Jiang, Z.S.; Pan, M. Correlation between postoperative early recurrence of hepatocellular carcinoma and mesenchymal circulating tumor cells in peripheral blood. J. Gastrointest. Surg. 2018, 22, 633-639. [CrossRef]

34. Sun, C.; Liao, W.; Deng, Z.; Li, E.; Feng, Q.; Lei, J.; Yuan, R.; Zou, S.; Mao, Y.; Shao, J.; et al. The diagnostic value of assays for circulating tumor cells in hepatocellular carcinoma: A meta-analysis. Medicine (Baltimore) 2017, 96, e7513. [CrossRef]

35. Yu, J.; Xiao, W.; Dong, S.; Liang, H.; Zhang, Z.; Zhang, B.; Huang, Z.; Chen, Y.; Zhang, W.; Lu, H.; et al. Effect of surgical liver resection on circulating tumor cells in patients with hepatocellular carcinoma. BMC Cancer 2018, 18, 835. [CrossRef]

36. Lin, H.C.; Liou, M.J.; Hsu, H.L.; Hsieh, J.C.; Chen, Y.A.; Tseng, C.P.; Lin, J.D. Combined analysis of circulating epithelial cells and serum thyroglobulin for distinguishing disease status of the patients with papillary thyroid carcinoma. Oncotarget 2016, 7, 17242-17253. [CrossRef]

37. Hsieh, J.C.; Lin, H.C.; Huang, C.Y.; Hsu, H.L.; Wu, T.M.; Lee, C.L.; Chen, M.C.; Wang, H.M.; Tseng, C.P. Prognostic value of circulating tumor cells with podoplanin expression in patients with locally advanced or metastatic head and neck squamous cell carcinoma. Head Neck 2015, 37, 1448-1455. [CrossRef]

38. Hsieh, C.H.; Lin, H.C.; Huang, S.B.; Hsueh, C.; Hsu, H.L.; Wang, H.M.; Wu, M.H.; Tseng, C.P.; Lin, J.D. Circulating epithelial cell enumeration facilitates the identification and follow-up of a patient with early stage papillary thyroid microcarcinoma: A case report. Clin. Chim. Acta. 2016, 454, 107-111. [CrossRef]

39. Li, Y.R.; Tseng, C.P.; Hsu, H.L.; Lin, H.C.; Chen, Y.A.; Chen, S.T.; Liou, M.J.; Lin, J.D. Circulating epithelial cells as potential biomarkers for detection of recurrence in patients of papillary thyroid carcinoma with positive serum anti-thyroglobulin antibody. Clin. Chim. Acta. 2018, 477, 74-80. [CrossRef]

40. Li, Y.M.; Xu, S.C.; Li, J.; Han, K.Q.; Pi, H.F.; Zheng, L.; Zuo, G.H.; Huang, X.B.; Li, H.Y.; Zhao, H.Z.; et al. Epithelial-mesenchymal transition markers expressed in circulating tumor cells in hepatocellular carcinoma patients with different stages of disease. Cell Death Dis. 2013, 4, e831. [CrossRef]

41. Lee, H.Y.; Yu, N.Y.; Lee, S.H.; Tsai, H.J.; Wu, C.C.; Cheng, J.C.; Chen, D.P.; Wang, Y.R.; Tseng, C.P. Podoplanin promotes cancer-associated thrombosis and contributes to the unfavorable overall survival in an ectopic xenograft mouse model of oral cancer. Biomed. J. 2019. [CrossRef]

42. Manion, K.; Frey, T. Apoptosis of cells in aged samples as detected by the ProCOUNT reagent. Cytometry 1996, 26, 317-322. [CrossRef]

43. Yu, H.K.; Lee, H.J.; Choi, H.N.; Ahn, J.H.; Choi, J.Y.; Song, H.S.; Lee, K.H.; Yoon, Y.; Yi, L.S.; Kim, J.S.; et al. Characterization of $\mathrm{CD} 45^{-} / \mathrm{CD} 31^{+} / \mathrm{CD} 105^{+}$circulating cells in the peripheral blood of patients with gynecologic malignancies. Clin. Cancer Res. 2013, 19, 5340-5350. [CrossRef] 
44. Fang, C.; Fan, C.; Wang, C.; Huang, Q.; Meng, W.; Yu, Y.; Yang, L.; Peng, Z.; Hu, J.; Li, Y.; et al. $\mathrm{CD} 133^{+} \mathrm{CD} 54^{+} \mathrm{CD} 44^{+}$circulating tumor cells as a biomarker of treatment selection and liver metastasis in patients with colorectal cancer. Oncotarget 2016, 7, 77389-77403. [CrossRef]

45. Kalinich, M.; Bhan, I.; Kwan, T.T.; Miyamoto, D.T.; Javaid, S.; LiCausi, J.A.; Milner, J.D.; Hong, X.; Goyal, L.; Sil, S.; et al. An RNA-based signature enables high specificity detection of circulating tumor cells in hepatocellular carcinoma. Proc. Natl. Acad. Sci. USA 2017, 114, 1123-1128. [CrossRef]

(C) 2020 by the authors. Licensee MDPI, Basel, Switzerland. This article is an open access article distributed under the terms and conditions of the Creative Commons Attribution (CC BY) license (http://creativecommons.org/licenses/by/4.0/). 